\title{
Estimates of the inner radii of non-overlapping domains
}

\author{
IRYNA DENEGA
}

(Presented by O. A. Dovgoshey)

Dedicated to the memory of Professor Bogdan Bojarski

\begin{abstract}
The paper is devoted to extremal problems of the geometric function theory of complex variable related with estimates of functionals defined on systems of non-overlapping domains. Till now, many such problems have not been solved, though some partial solutions are available. In the paper improved method is proposed for solving problems on extremal decomposition of the complex plane. The main results of the paper generalize and strengthening some known results in the theory of non-overlapping domains with free poles to the case of an arbitrary arrangement of systems of points on the complex plane.
\end{abstract}

2010 MSC. $30 \mathrm{C} 75$.

Key words and phrases. Inner radius of domain, non-overlapping domains, Green's function, transfinite diameter, theorem on minimizing of the area, the Cauchy inequality.

\section{Inequalities for the inner radii of symmetric non-overlapping domains on the unit circle}

Let $\mathbb{N}, \mathbb{R}$ be the sets of natural and real numbers, respectively, $\mathbb{C}$ be the complex plane, $\overline{\mathbb{C}}=\mathbb{C} \bigcup\{\infty\}$ be a one point compactification and $\mathbb{R}^{+}=(0, \infty)$. Let $r(B, a)$ be an inner radius of the domain $B \subset \overline{\mathbb{C}}$ relative to a point $a \in B$. The inner radius of the domain $B$ is connected with Green's generalized function $g_{B}(z, a)$ of the domain $B$ by the relations

$$
\begin{gathered}
g_{B}(z, a)=-\ln |z-a|+\ln r(B, a)+o(1), \quad z \rightarrow a, \\
g_{B}(z, \infty)=\ln |z|+\ln r(B, \infty)+o(1), \quad z \rightarrow \infty .
\end{gathered}
$$

Received 24.12.2018 
The system of points $A_{n}:=\left\{a_{k} \in \mathbb{C}, k=\overline{1, n}\right\}, n \in \mathbb{N}, n \geqslant 2$ is called $n$-radial, if $\left|a_{k}\right| \in \mathbb{R}^{+}$for $k=\overline{1, n}$ and

$$
0=\arg a_{1}<\arg a_{2}<\ldots<\arg a_{n}<2 \pi .
$$

Denote $a_{n+1}:=a_{1}, \alpha_{k}:=\frac{1}{\pi} \arg \frac{a_{k+1}}{a_{k}}, \alpha_{n+1}:=\alpha_{1}, k=\overline{1, n}, \sum_{k=1}^{n} \alpha_{k}=2$. Consider the following extremal problem.

Problem. For any fixed values of $\gamma \in(0, n]$ to find the maximum of the functional

$$
I_{n}(\gamma)=r^{\gamma}\left(B_{0}, 0\right) \prod_{k=1}^{n} r\left(B_{k}, a_{k}\right)
$$

where $B_{0}, B_{1}, B_{2}, \ldots, B_{n}, n \geqslant 2$, are mutually non-overlapping domains in $\overline{\mathbb{C}}$ and $B_{1}, \ldots, B_{n}$ are symmetric about the unit circle, $a_{0}=0,\left|a_{k}\right|=1$, $k=\overline{1, n}, a_{k} \in B_{k} \subset \overline{\mathbb{C}}, k=\overline{0, n}$.

This problem belongs to the class of extremal problems with free poles. Problems of this type have been studied in many papers (see, for example, [1-13]). In 1968 P. M. Tamrazov in the paper [10] first attracted the attention of experts to the study of the extremal problems associated with quadratic differentials with nonfixed poles possessing a definite freedom. And he solved a significant extremal problem of the geometric theory of functions of a complex variable with five free simple poles. In the works $[1,9]$ a very efficient method of separating transformation was developed with the help of which it was possible to solve some difficult problems with free poles on a circle.

Problem posed above in the case $\gamma=1$ was formulated as an open problem in the paper [1]. For $\gamma=1$ and $n \geqslant 2$ this problem was solved by L. V. Kovalev [3,4]. Namely, it was shown that under its conditions the inequality is true

$$
r\left(B_{0}, 0\right) \prod_{k=1}^{n} r\left(B_{k}, a_{k}\right) \leqslant r\left(D_{0}, 0\right) \prod_{k=1}^{n} r\left(D_{k}, d_{k}\right),
$$

where $d_{k}, D_{k}, k=\overline{0, n}$, are, respectively, poles and circular domains of the quadratic differential

$$
Q(w) d w^{2}=-\frac{w^{2 n}+2\left(n^{2}-1\right) w^{n}+1}{w^{2}\left(w^{n}-1\right)^{2}} d w^{2} .
$$

However, for the values of $\gamma \neq 1$, this problem has not been solved for a long time. Only in 2018 in the paper [5] it was solved for $n \geqslant 2$ and $\gamma \in(0,1)$. In the paper we propose a method which allows to obtained estimate of the maximum of the functional $I_{n}(\gamma)$ for this problem for all $n \geqslant 2$ and $\gamma \in(1, n]$. We obtain the following result. 
Theorem 1. Let $n \in \mathbb{N}, n \geqslant 2, \gamma \in(1, n]$. Then, for any system of different points $\left\{a_{k}\right\}_{k=1}^{n}$ of the unit circle and any mutually non-overlapping domains $B_{k}, a_{k} \in B_{k} \subset \overline{\mathbb{C}}, k=\overline{0, n}, a_{0}=0$, and $B_{k}, k=\overline{1, n}$, are symmetric about the unit circle $\left|a_{k}\right|=1$, the following inequality holds

$$
r^{\gamma}\left(B_{0}, 0\right) \prod_{k=1}^{n} r\left(B_{k}, a_{k}\right) \leqslant n^{-\frac{\gamma}{2}}\left(\prod_{k=1}^{n} r\left(B_{k}, a_{k}\right)\right)^{1-\frac{\gamma}{n}} .
$$

Proof. From the definition of the Green function we have

$$
r\left(B_{0}, 0\right)=r\left(B_{0}^{+}, \infty\right), \quad B^{+}=\left\{z ; \frac{1}{z} \in B\right\} .
$$

Let $E \subset \mathbb{C}$ be a compact infinite set of the complex plane. Let $d(E)$ be the transfinite diameter of a compact set $E \subset \mathbb{C}$. Then the following relation holds

$$
r\left(B_{0}, 0\right)=r\left(B_{0}^{+}, \infty\right)=\frac{1}{d\left(\overline{\mathbb{C}} \backslash B_{0}^{+}\right)} \leqslant \frac{1}{d\left(\bigcup_{k=1}^{n} \bar{B}_{k}^{+}\right)} .
$$

By virtue of the well-known Polya theorem $[6$, p. 28], [7, p. 34], the inequality

$$
\mu E \leqslant \pi d^{2}(E),
$$

where $\mu E$ denotes the Lebesgue measure of a compact set $E$, is valid. From whence, we get

$$
d(E) \geqslant\left(\frac{1}{\pi} \mu E\right)^{\frac{1}{2}} .
$$

Then relation (1.2) yields

$$
r\left(B_{0}, 0\right) \leqslant \frac{1}{d\left(\bigcup_{k=1}^{n} \bar{B}_{k}^{+}\right)} \leqslant \frac{1}{\sqrt{\frac{1}{\pi} \mu\left(\bigcup_{k=1}^{n} \bar{B}_{k}^{+}\right)}}=\left[\frac{1}{\pi} \sum_{k=1}^{n} \mu \bar{B}_{k}^{+}\right]^{-\frac{1}{2}} .
$$

For a bounded domain $B, a \in B$ we consider the class of all regular functions $\varphi(z), \varphi(a)=0, \varphi^{\prime}(a)=1$, given in the domain $B$, and the area of an image of the domain $B$ at the mapping by an arbitrary function $\varphi(z)$. It follows from the theorem of minimization of areas [7, p. 34] that

$$
\iint_{B}\left|\varphi^{\prime}(z)\right|^{2} d x d y \geqslant \pi r^{2}(B, a),
$$


where $r(B, a)$ is the inner radius of the domain $B$ with respect to the point $a$. Let us set $\varphi_{1}(z)=(z-a)$. Then relation (1.4) yields

$$
S(B)=\mu(B) \geqslant \pi r^{2}(B, a) .
$$

Inequality (1.3) implies directly that

$$
r\left(B_{0}, 0\right) \leqslant\left[\frac{1}{\pi} \sum_{k=1}^{n} \mu \bar{B}_{k}^{+}\right]^{-\frac{1}{2}} \leqslant\left[\frac{1}{\pi} \sum_{k=1}^{n} \mu B_{k}^{+}\right]^{-\frac{1}{2}} \leqslant\left[\sum_{k=1}^{n} r^{2}\left(B_{k}^{+}, a_{k}^{+}\right)\right]^{-\frac{1}{2}} .
$$

From whence, we get the inequality

$$
r\left(B_{0}, 0\right) \leqslant \frac{1}{\left[\sum_{k=1}^{n} r^{2}\left(B_{k}^{+}, a_{k}^{+}\right)\right]^{\frac{1}{2}}} .
$$

With regard for the relation

$$
r\left(B_{k}^{+}, a_{k}^{+}\right)=\frac{r\left(B_{k}, a_{k}\right)}{\left|a_{k}\right|^{2}}
$$

we arrive at the inequality

$$
r\left(B_{0}, 0\right) \leqslant\left[\frac{1}{\sum_{k=1}^{n} \frac{r^{2}\left(B_{k}, a_{k}\right)}{\left|a_{k}\right|^{4}}}\right]^{\frac{1}{2}} .
$$

This result and the assumption of Theorem 1 yield the relation

$$
r^{\gamma}\left(B_{0}, 0\right) \prod_{k=1}^{n} r\left(B_{k}, a_{k}\right) \leqslant \frac{\prod_{k=1}^{n} r\left(B_{k}, a_{k}\right)}{\left[\sum_{k=1}^{n} \frac{r^{2}\left(B_{k}, a_{k}\right)}{\left|a_{k}\right|^{4}}\right]^{\frac{\gamma}{2}}} .
$$

The Cauchy inequality yields automatically the relation

$$
\frac{1}{n} \sum_{k=1}^{n} \frac{r^{2}\left(B_{k}, a_{k}\right)}{\left|a_{k}\right|^{4}} \geqslant\left[\prod_{k=1}^{n} \frac{r^{2}\left(B_{k}, a_{k}\right)}{\left|a_{k}\right|^{4}}\right]^{\frac{1}{n}} .
$$

And using the equality

$$
\prod_{k=1}^{n}\left|a_{k}\right|=1
$$


we get easily

$$
\left[\sum_{k=1}^{n} \frac{r^{2}\left(B_{k}, a_{k}\right)}{\left|a_{k}\right|^{4}}\right]^{\frac{\gamma}{2}} \geqslant\left[n\left[\prod_{k=1}^{n} \frac{r^{2}\left(B_{k}, a_{k}\right)}{\left|a_{k}\right|^{4}}\right]^{\frac{1}{n}}\right]^{\frac{\gamma}{2}} \geqslant n^{\frac{\gamma}{2}}\left[\prod_{k=1}^{n} r\left(B_{k}, a_{k}\right)\right]^{\frac{\gamma}{n}}
$$

Eventually, from (1.5) we obtain

$$
r^{\gamma}\left(B_{0}, 0\right) \prod_{k=1}^{n} r\left(B_{k}, a_{k}\right) \leqslant n^{-\frac{\gamma}{2}}\left[\prod_{k=1}^{n} r\left(B_{k}, a_{k}\right)\right]^{1-\frac{\gamma}{n}} .
$$

Thus Theorem 1 is proved.

Remark 1. If $\gamma=n$, then from Theorem 1 the following inequality holds

$$
r^{n}\left(B_{0}, 0\right) \prod_{k=1}^{n} r\left(B_{k}, a_{k}\right) \leqslant n^{-\frac{n}{2}}
$$

Using Theorem 5.1.1 [8] from Theorem 1 we have the following statement.

Corollary 1. Let $n \in \mathbb{N}, n \geqslant 2, \gamma \in(1, n]$. Then, for any system of different points $\left\{a_{k}\right\}_{k=1}^{n}$ of the unit circle and any mutually non-overlapping domains $B_{k}, a_{k} \in B_{k} \subset \overline{\mathbb{C}}, k=\overline{0, n}, a_{0}=0$, and $B_{k}, k=\overline{1, n}$, are symmetric about the unit circle $\left|a_{k}\right|=1$, the following inequality holds

$$
r^{\gamma}\left(B_{0}, 0\right) \prod_{k=1}^{n} r\left(B_{k}, a_{k}\right) \leqslant 2^{(n-\gamma)} \cdot n^{-\frac{\gamma}{2}} \cdot\left(\prod_{k=1}^{n} \alpha_{k}\right)^{1-\frac{\gamma}{n}}
$$

From Theorem 6.11 [9, p.172] we obtain the following inequality.

Corollary 2. Let $n \in \mathbb{N}, n \geqslant 2, \gamma \in(1, n]$. Then, under the conditions of the Corollary 1 the following inequality holds

$$
r^{\gamma}\left(B_{0}, 0\right) \prod_{k=1}^{n} r\left(B_{k}, a_{k}\right) \leqslant n^{-\frac{\gamma}{2}}\left(\frac{4}{n}\right)^{n-\gamma}
$$

Let

$$
I_{n}^{0}(\gamma)=r^{\gamma}\left(D_{0}, d_{0}\right) \cdot \prod_{k=1}^{n} r\left(D_{k}, d_{k}\right)
$$

where $D_{k}$ and $d_{k}, k=\overline{0, n}$ are the circular domains and, respectively, the poles of the quadratic differential

$$
Q(w) d w^{2}=-\frac{\gamma w^{2 n}+2\left(n^{2}-\gamma\right) w^{n}+\gamma}{w^{2}\left(w^{n}-1\right)^{2}} d w^{2}
$$


From [1-5] we have

$$
I_{n}^{0}(\gamma)=\left(\frac{4}{n}\right)^{n} \frac{\left(\frac{2 \gamma}{n^{2}}\right)^{\frac{\gamma}{n}}}{\left|1-\frac{2 \gamma}{n^{2}}\right|^{\frac{n}{2}+\frac{\gamma}{n}}}\left|\frac{n-\sqrt{2 \gamma}}{n+\sqrt{2 \gamma}}\right|^{\sqrt{2 \gamma}} .
$$

We made a comparative analysis of estimate of the maximum of functional $I_{n}(\gamma)$ obtained in Theorem 1 and the estimate $I_{n}^{0}(\gamma)$ when $\gamma=n$ for $n=\overline{2,10}$ (see table below).

\begin{tabular}{|c|c|c|c|c|}
\hline$n$ & $I_{n}^{0}(n)$ & $n^{-\frac{n}{2}}$ & $n^{-\frac{n}{2}}-I_{n}^{0}(n)$ & $\frac{n^{-\frac{n}{2}}-I_{n}^{0}(n)}{I_{n}^{0}(n)}$ \\
\hline 2 & 0,2500000000 & 0,5000000000 & 0,2500000000 & 1,0000000000 \\
\hline 3 & 0,0897092419 & 0,1924500897 & 0,1027408478 & 1,1452649211 \\
\hline 4 & 0,0273370712 & 0,0625000000 & 0,0351629288 & 1,2862727153 \\
\hline 5 & 0,0070194764 & 0,0178885438 & 0,0108690674 & 1,5484156742 \\
\hline 6 & 0,0015467153 & 0,0046296296 & 0,0030829143 & 1,9932008548 \\
\hline 7 & 0,0002977029 & 0,0011019372 & 0,0008042344 & 2,7014669766 \\
\hline 8 & 0,0000508051 & 0,0002441406 & 0,0001933355 & 3,8054339019 \\
\hline 9 & 0,0000077826 & 0,0000508053 & 0,0000430227 & 5,5280820279 \\
\hline 10 & 0,0000010811 & 0,0000100000 & 0,0000089189 & 8,2502515613 \\
\hline
\end{tabular}

\section{Inequalities for the inner radii of non-overlapping domains on the complex plane}

From method of proof of the Theorem 1 we can obtain estimates of the next functional

$$
J_{n}(\gamma)=\left[r\left(B_{0}, 0\right) r\left(B_{\infty}, \infty\right)\right]^{\gamma} \prod_{k=1}^{n} r\left(B_{k}, a_{k}\right),
$$

considered, for example, in the papers [1, $8,9,13]$, in which for $J_{n}(\gamma)$ in particular cases for some values of $\gamma$, the following inequality was established

$$
J_{n}(\gamma) \leqslant\left(\frac{4}{n}\right)^{n} \frac{\left(\frac{4 \gamma}{n^{2}}\right)^{\frac{2 \gamma}{n}}}{\left|1-\frac{4 \gamma}{n^{2}}\right|^{\frac{2 \gamma}{n}+\frac{n}{2}}}\left|\frac{n-2 \sqrt{\gamma}}{n+2 \sqrt{\gamma}}\right|^{2 \sqrt{\gamma}}
$$

Equality in this inequality is achieved when $0, \infty, a_{k}$ and $B_{0}, B_{\infty}, B_{k}$, $k=\overline{1, n}$, are, respectively, poles and circular domains of the quadratic differential

$$
Q(w) d w^{2}=-\frac{\gamma w^{2 n}+\left(n^{2}-2 \gamma\right) w^{n}+\gamma}{w^{2}\left(w^{n}-1\right)^{2}} d w^{2}
$$


Namely, the following result holds.

Theorem 2. Let $n \in \mathbb{N}, n \geqslant 2, \gamma \in\left(0, \frac{n+2}{2}\right]$. Then, for any fixed system of different points $A_{n}=\left\{a_{k}\right\}_{k=1}^{n} \in \mathbb{C} /\{0, \infty\}$ and any mutually non-overlapping domains $B_{0}, B_{\infty}, B_{k}, a_{0}=0 \in B_{0} \subset \overline{\mathbb{C}}, \infty \in B_{\infty} \subset \overline{\mathbb{C}}$, $a_{k} \in B_{k} \subset \overline{\mathbb{C}}, k=\overline{1, n}$, the following inequality holds

$$
J_{n}(\gamma) \leqslant(n+1)^{-\frac{n+1}{n+2} \gamma}\left(\prod_{k=1}^{n} r\left(B_{k}, a_{k}\right)\right)^{1-\frac{2 \gamma}{n+2}}\left(\prod_{k=1}^{n}\left|a_{k}\right|\right)^{\frac{2 \gamma}{n+2}}
$$

Proof. Using inequalities (1.2), (1.3) and (1.4), we have

$$
\begin{gathered}
r\left(B_{0}, 0\right) \leqslant \frac{1}{\left[r^{2}\left(B_{\infty}^{+}, 0\right)+\sum_{k=1}^{n} r^{2}\left(B_{k}^{+}, a_{k}^{+}\right)\right]^{\frac{1}{2}}} \\
=\frac{1}{\left[r^{2}\left(B_{\infty}, \infty\right)+\sum_{k=1}^{n} \frac{r^{2}\left(B_{k}, a_{k}\right)}{\left|a_{k}\right|^{4}}\right]^{\frac{1}{2}}}, \\
r\left(B_{\infty}, \infty\right) \leqslant \frac{1}{\left[r^{2}\left(B_{0}, 0\right)+\sum_{k=1}^{n} r^{2}\left(B_{k}, a_{k}\right)\right]^{\frac{1}{2}}} .
\end{gathered}
$$

Taking into account the Cauchy inequality

$$
\frac{1}{n+1}\left(r^{2}\left(B_{0}, 0\right)+\sum_{k=1}^{n} r^{2}\left(B_{k}, a_{k}\right)\right) \geqslant\left[r^{2}\left(B_{0}, 0\right) \prod_{k=1}^{n} r^{2}\left(B_{k}, a_{k}\right)\right]^{\frac{1}{n+1}} .
$$

Then

$$
\left(r^{2}\left(B_{0}, 0\right)+\sum_{k=1}^{n} r^{2}\left(B_{k}, a_{k}\right)\right)^{\frac{1}{2}} \geqslant(n+1)^{\frac{1}{2}}\left[r\left(B_{0}, 0\right) \prod_{k=1}^{n} r\left(B_{k}, a_{k}\right)\right]^{\frac{1}{n+1}} .
$$

Analogically,

$$
\left(r^{2}\left(B_{\infty}, \infty\right)+\sum_{k=1}^{n} \frac{r^{2}\left(B_{k}, a_{k}\right)}{\left|a_{k}\right|^{4}}\right)^{\frac{1}{2}} \geqslant(n+1)^{\frac{1}{2}}\left[r\left(B_{\infty}, \infty\right) \prod_{k=1}^{n} \frac{r\left(B_{k}, a_{k}\right)}{\left|a_{k}\right|^{2}}\right]^{\frac{1}{n+1}}
$$

Thus,

$$
r\left(B_{\infty}, \infty\right) \leqslant \frac{1}{(n+1)^{\frac{1}{2}}\left(r\left(B_{0}, 0\right)\right)^{\frac{1}{n+1}}\left(\prod_{k=1}^{n} r\left(B_{k}, a_{k}\right)\right)^{\frac{1}{n+1}}}
$$




$$
r\left(B_{0}, 0\right) \leqslant \frac{\left(\prod_{k=1}^{n}\left|a_{k}\right|\right)^{\frac{2}{n+1}}}{(n+1)^{\frac{1}{2}}\left(r\left(B_{\infty}, \infty\right)\right)^{\frac{1}{n+1}}\left(\prod_{k=1}^{n} r\left(B_{k}, a_{k}\right)\right)^{\frac{1}{n+1}}} .
$$

Further, we obtain the relations

$$
\begin{gathered}
r\left(B_{0}, 0\right) r\left(B_{\infty}, \infty\right) \\
\leqslant \frac{\left(\prod_{k=1}^{n}\left|a_{k}\right|\right)^{\frac{2}{n+1}}}{(n+1)\left(r\left(B_{0}, 0\right) r\left(B_{\infty}, \infty\right)\right)^{\frac{1}{n+1}}\left(\prod_{k=1}^{n} r\left(B_{k}, a_{k}\right)\right)^{\frac{2}{n+1}}}, \\
\left(r\left(B_{0}, 0\right) r\left(B_{\infty}, \infty\right)\right)^{1+\frac{1}{n+1}} \leqslant \frac{\left(\prod_{k=1}^{n}\left|a_{k}\right|\right)^{\frac{2}{n+1}}}{(n+1)\left(\prod_{k=1}^{n} r\left(B_{k}, a_{k}\right)\right)^{\frac{2}{n+1}}}, \\
r\left(B_{0}, 0\right) r\left(B_{\infty}, \infty\right) \leqslant \frac{\left(\prod_{k=1}^{n}\left|a_{k}\right|\right)^{\frac{2}{n+2}}}{(n+1)^{\frac{n+1}{n+2}}\left(\prod_{k=1}^{n} r\left(B_{k}, a_{k}\right)\right)^{\frac{2}{n+2}}},
\end{gathered}
$$

from which inequality (2.1) of the Theorem 2 follows

$$
\begin{gathered}
J_{n}(\gamma) \leqslant \frac{\prod_{k=1}^{n} r\left(B_{k}, a_{k}\right)\left(\prod_{k=1}^{n}\left|a_{k}\right|\right)^{\frac{2}{n+2}}}{(n+1)^{\frac{n+1}{n+2} \gamma}\left(\prod_{k=1}^{n} r\left(B_{k}, a_{k}\right)\right)^{\frac{2 \gamma}{n+2}}} \\
=(n+1)^{-\frac{n+1}{n+2} \gamma}\left(\prod_{k=1}^{n} r\left(B_{k}, a_{k}\right)\right)^{1-\frac{2 \gamma}{n+2}}\left(\prod_{k=1}^{n}\left|a_{k}\right|\right)^{\frac{2 \gamma}{n+2}} .
\end{gathered}
$$

Remark 2. If $\gamma=\frac{n+2}{2}$ and $\prod_{k=1}^{n}\left|a_{k}\right| \leqslant 1$ then from Theorem 2 the following inequality holds

$$
J_{n}(\gamma) \leqslant(n+1)^{-\frac{n+1}{2}} .
$$


From Theorem 2 we have the following statements.

Corollary 3. Let $n \in \mathbb{N}, n \geqslant 2, \gamma \in\left(0, \frac{n+2}{2}\right]$. Then, for any system of different points $\left\{a_{k}\right\}_{k=1}^{n}$ of the unit circle and any mutually nonoverlapping domains $B_{0}, B_{\infty}, B_{k}, a_{0}=0 \in B_{0} \subset \overline{\mathbb{C}}, \infty \in B_{\infty} \subset \overline{\mathbb{C}}$, $a_{k} \in B_{k} \subset \overline{\mathbb{C}}, k=\overline{1, n}$, the following inequality holds

$$
J_{n}(\gamma) \leqslant(n+1)^{-\frac{n+1}{n+2} \gamma}\left(2^{n} \prod_{k=1}^{n} \alpha_{k}\right)^{1-\frac{2 \gamma}{n+2}} .
$$

Corollary 4. Let $n \in \mathbb{N}, n \geqslant 2, \gamma \in\left(0, \frac{n+2}{2}\right]$. Then, under the conditions of the Corollary 3 the following inequality holds

$$
J_{n}(\gamma) \leqslant(n+1)^{-\frac{n+1}{n+2} \gamma}\left(\frac{4}{n}\right)^{n-\frac{2 n \gamma}{n+2}} .
$$

From Theorem 2 on condition $B_{0} \subset U$, we obtain a result giving some estimate in the problem stated in the paper of G. P. Bakhtina [2].

Theorem 3. Let $n \in \mathbb{N}, n \geqslant 2, \gamma \in\left(0, \frac{n+2}{2}\right]$ and $B_{0} \subset U$. Then, for any system of different points $\left\{a_{k}\right\}_{k=1}^{n}$ of the unit circle and any mutually non-overlapping domains $B_{k}, a_{k} \in B_{k} \subset \overline{\mathbb{C}}, k=\overline{0, n}, a_{0}=0$, and $B_{k}$, $k=\overline{1, n}$, are symmetric about the unit circle $\left|a_{k}\right|=1$, the following inequality holds

$$
\begin{gathered}
r^{2 \gamma}\left(B_{0}, 0\right) \prod_{k=1}^{n} r\left(B_{k}, a_{k}\right) \\
\leqslant(n+1)^{-\frac{\gamma(n+1)}{n+2}}\left(\prod_{k=1}^{n} r\left(B_{k}, a_{k}\right)\right)^{1-\frac{2 \gamma}{n+2}}\left(\prod_{k=1}^{n}\left|a_{k}\right|\right)^{\frac{2 \gamma}{n+2}} .
\end{gathered}
$$

Let $p, q \in \mathbb{N}$. A set of points $A_{p, q}:=\left\{a_{k, s} \in \mathbb{C}: k=\overline{1, p}, s=\overline{1, q}\right\}$ is called $(p, q)$-radial system, if for all $k=\overline{1, p}$ and $s=\overline{1, q}$ the relations hold

$$
\begin{aligned}
& 0<\left|a_{k, 1}\right|<\ldots<\left|a_{k, q}\right|<\infty \\
& \arg a_{k, 1}=\arg a_{k, 2}=\ldots=\arg a_{k, q}=: \theta_{k}=: \theta_{k}\left(A_{p, q}\right) ; \\
& 0=\theta_{1}<\theta_{2}<\ldots<\theta_{p}<\theta_{p+1}:=2 \pi .
\end{aligned}
$$

Then, from method of proofs of the above presented theorems we obtain the following corollaries for $A_{p, q}$-radial systems points.

Corollary 5. Let $p, q \in \mathbb{N}, p \geqslant 2, \gamma \in(0, p q]$. Then, for any fixed $(p, q)$-radial system of points $A_{p, q}=\left\{a_{k, s}\right\}, k=\overline{1, p}, s=\overline{1, q}$, and any 
mutually non-overlapping domains $B_{0}, B_{k, s}, a_{k, s} \in B_{k, s} \subset \overline{\mathbb{C}}, k=\overline{1, p}$, $s=\overline{1, q}, a_{0}=0 \in B_{0} \subset \overline{\mathbb{C}}$, the inequality holds

$$
\begin{gathered}
r^{\gamma}\left(B_{0}, 0\right) \prod_{k=1}^{p} \prod_{s=1}^{q} r\left(B_{k, s}, a_{k, s}\right) \\
\leqslant(p q)^{-\frac{\gamma}{2}}\left(\prod_{k=1}^{p} \prod_{s=1}^{q} r\left(B_{k, s}, a_{k, s}\right)\right)^{1-\frac{\gamma}{p q}}\left(\prod_{k=1}^{p} \prod_{s=1}^{q}\left|a_{k, s}\right|\right)^{\frac{2 \gamma}{p q}} .
\end{gathered}
$$

Corollary 6. Let $p, q \in \mathbb{N}, p \geqslant 2, \gamma \in\left(0, \frac{p q+2}{2}\right]$. Then, for any fixed $(p, q)$-radial system of points $A_{p, q}=\left\{a_{k, s}\right\}, k=\overline{1, p}, s=\overline{1, q}$, and any mutually non-overlapping domains $B_{0}, B_{\infty}, B_{k, s}, a_{k, s} \in B_{k, s} \subset \overline{\mathbb{C}}$, $k=\overline{1, p}, s=\overline{1, q}, a_{0}=0 \in B_{0} \subset \overline{\mathbb{C}}, \infty \in B_{\infty} \subset \overline{\mathbb{C}}$, the inequality holds

$$
\begin{gathered}
{\left[r\left(B_{0}, 0\right) r\left(B_{\infty}, \infty\right)\right]^{\gamma} \prod_{k=1}^{p} \prod_{s=1}^{q} r\left(B_{k, s}, a_{k, s}\right)} \\
\leqslant(p q+1)^{-\frac{p q+1}{p q+2} \gamma}\left(\prod_{k=1}^{p} \prod_{s=1}^{q} r\left(B_{k, s}, a_{k, s}\right)\right)^{1-\frac{2 \gamma}{p q+2}}\left(\prod_{k=1}^{p} \prod_{s=1}^{q}\left|a_{k, s}\right|\right)^{\frac{2 \gamma}{p q+2}} .
\end{gathered}
$$

\section{Acknowlegements}

The author thanks the Prof. Alexander Bakhtin for posing problems, careful analysis of this work and remarks.

\section{References}

[1] V. N. Dubinin, Symmetrization method in geometric function theory of complex variables // Successes Mat. Science, 49 (1994), No. 1 (295), 3-76 (in Russian); translation in Russian Math. Surveys, 1 (1994), 1-79.

[2] G. P. Bakhtina, On conformal radii of symmetric non-overlapping domains // Modern problems of the real and complex analysis, Kiev, Institute of Mathematics of the Academy of Sciences of USSR, 1984, 21-27 (in Russian).

[3] L. V. Kovalev, On the inner radii of symmetric nonoverlapping domains // Izv. Vyssh. Uchebn. Zaved. Mat., (2000), No. 6, 80-81 (in Russian).

[4] L. V. Kovalev, On three disjoint domains // Dal'nevost. Mat. Zh., 1 (2000), No. 1, 3-7 (in Russian).

[5] Ya. V. Zabolotnii, L. V. Vyhivska, On a product of the inner radii of symmetric multiply connected domains // Ukrainian Mathematical Bulletin, 14 (2017), No. 3, 441-451; translation in J. Math. Sci., 231 (2018), No. 1, 101-109. 
[6] G. Polya, G. Szego, Isoperimetric inequalities in mathematical physics, M, Fizmatgiz, 1962 (in Russian).

[7] G. M. Goluzin, Geometric theory of functions of a complex variable // Amer. Math. Soc. Providence, R.I., 1969.

[8] A. K. Bakhtin, G. P. Bakhtina, Yu. B. Zelinskii, Topological-algebraic structures and geometric methods in complex analysis // Zb. prats of the Inst. of Math. of NASU, 2008 (in Russian).

[9] V. N. Dubinin, Condenser capacities and symmetrization in geometric function theory, Birkhäuser/Springer, Basel, 2014.

[10] P. M. Tamrazov, Extremal conformal mappings and poles of quadratic differentials // Mathematics of the USSR-Izvestiya, 2 (1968), No. 5, 987-996.

[11] A. K. Bakhtin, G. P. Bakhtina, I. V. Denega, Extremal decomposition of a complex plane with fixed poles // Zb. pr. In-t matem. of NAS of Ukraine, 14 (2017), No. 1, $34-38$.

[12] A. K. Bakhtin, Separating transformation and extremal problems on nonoverlapping simply connected domains // Ukrainian Mathematical Bulletin, 14 (2017), No. 4, 456-471 (in Russian); translation in J. Math. Sci. 234 (2018), No. 1, 1-13.

[13] G. V. Kuzmina, Problems on extremal decomposition of the riemann sphere // Notes scientific. Sem. Leningr. Dep. of Math. Inst. AN USSR., 276 (2001), 253275 (in Russian); translation in J. Math. Sci. (N.Y.), 118 (2003), No. 1, 48804894 .

\section{CONTACT INFORMATION}

Iryna Denega

Institute of mathematics of National

Academy of Sciences of Ukraine,

Kyiv, Ukraine

E-Mail: iradenega@gmail.com 